\title{
Avaliação de opções de swing em contratos de gás natural usando um modelo de dois fatores
}

\author{
Carlos Patricio Samanez, Letícia de Almeida Costa ${ }^{\mathrm{b} *}$ \\ acps@puc-rio.br, PUC-Rio, Brasil \\ b*leticiaalmeidacosta@gmail.com, PUC-Rio, Brasil
}

\begin{abstract}
Resumo
No mercado de gás natural (GN), muitos contratos incorporam flexibilidades no volume, conhecidas como opções de swing, as quais concedem ao titular a opção de exercer o direito de receber volumes maiores ou menores, de acordo com o mercado. Neste artigo, o preço do GN é a principal fonte de incerteza e foi modelado com um processo estocástico seguindo o modelo de dois fatores de Schwartz e Smith (2000) ao qual foi incorporada a sazonalidade trimestral. Para estimar os preços à vista do GN, usando os preços dos contratos futuros do Henry Hub, implementouse o filtro de Kalman. 0 apreçamento da opção foi realizado através do modelo de árvore binomial bivariável desenvolvido por Hahn e Dyer (2011). 0 valor da opção de swing foi positivo nos dois casos analisados, mostrando que essa opção tem valor e deve ser analisada para inclusão nos contratos de GN. As características do contrato analisado foram as mesmas especificadas em Jaillet, Ronn e Tompaidis (2004).
\end{abstract}

\section{Palavras-chave}

Finanças. Derivativos. Precificação de contratos. Processos estocásticos.

\section{Introdução}

0 mercado de gás natural (GN), em geral, tem suas vendas atreladas a contratos de longo prazo e com baixa liquidez. As incertezas futuras que possam ocorrer na economia ou no setor de energia, ao longo da vida de um contrato, fazem com que os agentes incorporem flexibilidades no contrato de modo a se beneficiarem com mudanças não previstas nos preços de mercado ou demanda. No setor de energia, em particular do GN, essas flexibilidades podem ser com relação ao volume de GN a ser entregue, conhecidas como opções de swing. Sujeitos a restrições, esses contratos permitem ao titular o direito de receber volumes maiores ou menores de GN em relação ao inicialmente contratado, de acordo com a evolução dos preços, indicadores econômicos e da demanda. Ou seja, há necessidade de flexibilidades para responder eficientemente a mudanças do ambiente, não antecipadas no momento da contratação. Essas opções fazem parte das chamadas opções exóticas, cujas características são específicas, distinguindo-se das opções usuais.
Um dos aspectos importantes na valoração dos contratos é determinar de que forma são tratadas as incertezas. Na maioria dos estudos, assume-se que os fatores de incerteza seguem um determinado processo estocástico. No presente trabalho, o modelo de dois fatores de Schwartz e Smith (2000) para preços de commodities é usado para explorar a dinâmica do mercado de GN, com base na série histórica dos preços do Henry Hub Natural Gas Spot Prices. Nesse modelo, o preço à vista de uma commodity pode ser decomposto em dois fatores estocásticos, o preço de equilíbrio de longo prazo e as variações de curto prazo dos preços. Como em geral as commodities possuem sazonalidade em termos de preço, neste trabalho será adicionado ao modelo de Schwartz e Smith (2000) um componente sazonal.

Com base nos argumentos, análises e resultados encontrados nos principais trabalhos que avaliam contratos de GN, tais como os de Jaillet, Ronn e Tompaidis (2004), Figueroa (2006), Haff, Lindqvist e Løland (2007), Adland, Jia e Lu (2008), Cartea e 
Williams (2008), Hambly, Howison e Kluge (2008), Guigues, Sagatizabal e Zubelli (2010), Zhang e Oosterlee (2010) e Simões et al. (2011), foi inicialmente selecionado o modelo de Hull e White (1994b) para o apreçamento de opções de swing. A implementação do modelo é feita através de uma árvore trinomial para o modelo de dois fatores. Após ser feita uma análise baseada nos argumentos e análises de Park (2003), Parsons (2007), Brigo e Mercurio (2010) e Baldvinsdóttir e Palmborg (2011), concluiu-se que o modelo de Hull e White (1994b) para dois fatores se aplica a processos de reversão à média, mas não pode ser estendido para o modelo de dois fatores de Schwartz e Smith (2000). Do mesmo modo, árvores trinomiais sugeridas por Clewlow e Strickland (1999) e Tseng e lin (2007) também não se adaptam ao modelo de Schwartz e Smith (2000). Essa conclusão foi confirmada por Hahn e Dyer (2011), que apresentaram a mesma dificuldade concluindo na adoção de árvores binomiais baseadas em Boyle (1988) e Nelson e Ramaswamy (1990). Portanto, para o cálculo da opção, neste trabalho adotou-se o modelo discreto de Hahn e Dyer (2011) de árvores binomiais para dois fatores estocásticos.

Devido à não padronização dos contratos de GN no mercado brasileiro, foram avaliadas as opções de swing, mantendo as características básicas do contrato analisado por Jaillet, Ronn e Tompaidis (2004). 0 artigo avalia as flexibilidades sem a necessidade de uma matemática complexa, mas considerando os aspectos relevantes ao mercado de energia no Brasil.

0 estudo é estruturado em seis partes. A seção 2 contextualiza o mercado de GN de forma global e no Brasil. Na terceira seção, são apresentados os principais modelos estocásticos de dois fatores para preços de commodities e detalha o modelo de dois fatores de Schwartz e Smith (2000). A seção 4 trata das principais aproximações dos processos estocásticos através de árvores binomiais. Na seção 5, se faz uma exposição das principais características das opções de swing. A sexta seção explica detalhes dos contratos de GN com opções de swing. Também são detalhadas as variáveis do modelo, definidos os parâmetros e realizados os cálculos necessários para valorar a opção de swing, mostrando os principais resultados. Na seção 7 são expostos os resultados e na seção 8 são descritas as conclusões.

\section{0 mercado global de gás natural}

As transações comerciais que visam o fornecimento de GN implicam em um complexo conjunto de relações ao longo da cadeia de fornecimento. A produção de GN se inicia nos poços, resultado de investimentos exploratórios de alto risco. Em seguida, o gás é transportado por gasodutos e distribuído aos consumidores finais. Basicamente o $\mathrm{GN}$ pode ser transportado de duas formas diferentes, através de dutos e/ou de navios carregadores de gás natural liquefeito (GNL). Historicamente, o mercado de gás estruturou-se em função das características físicas do seu transporte. Os elevados custos de transporte do GN contribuíram negativamente para o desenvolvimento de um mercado global do combustível. Nas décadas de 1980 e 1990, o desenvolvimento de novas tecnologias de liquefação e transporte de GNL permitiu o aumento da flexibilidade do mercado, diversificando as rotas de comércio internacional do GN e ampliando o número de agentes no mercado (MATHIAS, 2008). Tornou-se viável economicamente levar essa commodity para vários destinos, principalmente para aqueles países que não possuem autossuficiência na produção e para aqueles que estão mudando sua matriz energética para fontes mais limpas, comparada ao carvão e ao petróleo.

0 desenvolvimento de técnicas para a extração de GN a partir do xisto (shale gas), que viabilizou economicamente esse tipo de produção e aumentou consideravelmente as reservas norte-americanas de GN, mudou bruscamente as previsões e perspectivas para o mercado americano. A exploração de GN utilizando essa nova técnica vem crescendo e as previsões de redução de volumes de sua importação se tornam cada vez mais realistas, afetando completamente o mercado energético. 0 potencial impacto da produção de gás de xisto sobre a dinâmica do comércio internacional de GN se reflete no descolamento dos preços no Henry $H u b$ em relação aos preços do petróleo. Isso tem ocorrido a partir de 2009, quando o preço do petróleo reverteu sua tendência de queda (GÁS NET, 2012).

\subsection{O mercado de gás natural no Brasil}

No Brasil, o mercado de GN vem apresentando um crescimento inédito nos últimos anos, mas seu desenvolvimento recente ocorreu em um contexto de relativa escassez de gás nacional. As reservas e a produção brasileira eram modestas, e em sua maioria proveniente de campos de gás associado pertencentes à Petrobras. A difusão do GN no país só se alavancou com o contrato de importação da Bolívia, o que viabilizou a construção do gasoduto Bolívia-Brasil (Gasbol). A construção do Gasbol teve início em 1997 e o duto entrou em operação em 1999 (AGÊNCIA..., 2001). A partir de 2000 , nota-se um crescimento significativo do mercado de GN graças à construção do Gasbol, o que permitiu complementar a produção nacional rapidamente e em grandes volumes.

As descobertas do pré-sal apresentam um grande potencial para produção de gás. Em média, os campos 
de óleo descobertos na área do pré-sal da Bacia de Santos contêm 20\% de GN. Além das descobertas no pré-sal, a exploração nas bacias de São Francisco (MG), Solimões (AM) e Parnaíba (MA) vem apontando um grande potencial produtivo para o GN. No caso dessas três bacias, o potencial produtivo é de gás não associado (PETROBRAS, 2012). Portanto, a produção somente se viabilizará caso haja mercado capaz de pagar um preço que possa cobrir os custos de produção e transporte. $\mathrm{Na}$ indústria de $\mathrm{GN}$, os investimentos e transporte só se viabilizam se houver mercado garantido para esse gás. Os investidores buscam assinar contratos de venda da produção futura do gás para depois injetar recursos na produção e transporte. Isso é necessário porque o GN não é uma commodity que pode ser transportada e comercializada para qualquer mercado. Os investimentos em transporte já definem onde e quem irá comprar o GN (BRASIL, 2011).

Tendo em vista o atual contexto energético do país é de se esperar uma participação cada vez maior do GN na matriz energética nacional (AGÊNCIA..., 2012). Tudo indica que a ampliação do mercado é uma tendência. Essa consolidação do GN na realidade do país é uma das motivações para se estudar e buscar flexibilidades nos contratos de GN negociados no Brasil.

Na comercialização da produção de GN prevalecem contratos de longo prazo com baixa liquidez, ou seja, contratos de alto risco cujas flexibilidades a serem incorporadas devem levar em consideração a previsão dos preços do GN, ao longo da vida útil do contrato. Para isso, torna-se necessário especificar um modelo para a previsão do preço do GN. A próxima seção apresenta modelos estocásticos de preços, detalhando o modelo de dois fatores de Schwartz e Smith (2000).

\section{Modelos de dois fatores}

Um dos primeiros trabalhos que trataram da modelagem de preços de commodities com base em processos estocásticos de preços foi realizado por Brennan e Schwartz (1985). Os autores apresentaram um modelo de fator único para projetar o preço futuro do cobre. Os trabalhos com modelos de preços de commodities, usando mais do que um único fator estocástico, começaram com Gibson e Schwartz (1990). Os autores desenvolveram um modelo de dois fatores para projetar os preços em contratos futuros de petróleo, em que os fatores de incerteza são o preço à vista e o retorno de conveniência. 0 processo estocástico usado para o preço do petróleo foi o Movimento Geométrico Browniano (MGB) e, para o retorno de conveniência, o Movimento de Reversão à Média (MRM).
Posteriormente, Schwartz (1997) analisou o comportamento estocástico dos preços das commodities, comparando-os por meio do uso de três modelos. 0 primeiro modelo considerou apenas um fator estocástico: o preço à vista da commodity que segue um MRM. 0 segundo modelo considerou dois fatores estocásticos: o preço à vista que segue um MGB, e o retorno de conveniência que evolui de acordo a um MRM. 0 terceiro modelo agrega a taxa de juros que segue um MRM. Os resultados evidenciaram que o modelo de um fator tem um desempenho fraco. Os modelos de dois e três fatores apresentaram desempenhos equivalentes, porém o autor observou que a taxa de juros agrega pouca informação e melhoria frente ao segundo modelo.

Já Schwartz e Smith (2000) apresentaram um modelo de dois fatores para analisar o comportamento dos preços das commodities e comparar com o modelo de Gibson e Schwartz (1990). Nesse modelo, o logaritmo do preço à vista é decomposto em dois fatores estocásticos correlacionados: o primeiro é modelado segundo um MRM e é relacionado às variações de curto prazo nos preços, e o segundo é modelado de acordo a um Movimento Aritmético Browniano (MAB) e é relacionado aos preços de equilíbrio. Os autores usaram na análise contratos futuros de petróleo, a mesma base de dados de Schwartz (1997).

Manoliu e Tompaidis (2000) usaram o modelo de Schwartz e Smith (2000) para analisar o comportamento dos preços do GN e incluíram uma função determinística para a sazonalidade. Os parâmetros de reversão encontrados foram significantes. Comparando os modelos de um e de dois fatores, os autores mostraram que o modelo de dois fatores permite melhor ajuste que o modelo de um fator.

Lucia e Schwartz (2001) apresentaram um caso sobre os derivativos no setor de energia elétrica, usando os modelos de um e de dois fatores. No modelo de um fator é usado o MRM, já no modelo de dois fatores são usados os MRM e o MGB. Em ambos os modelos foi incluída uma componente sazonal com variáveis dummies e funções senoidais. Os resultados mostraram que os parâmetros de sazonalidade são significantes nos preços de energia elétrica.

Sørensen (2002) apresentou um estudo sobre o comportamento estocástico dos preços futuros de commodities agrícolas, através do modelo de dois fatores de Schwartz e Smith (2000) introduzindo uma sazonalidade modelada por uma combinação linear de funções trigonométricas com frequências sazonais. Os resultados mostraram que as componentes sazonais acarretaram um pico de preços dois a três 
meses antes do período de colheita, atingindo valores mínimos no fim da safra. Esses padrões se justificam para manter o equilíbrio entre a oferta e a demanda.

Um modelo de multifatores foi proposto por Cortazar e Naranjo (2006) para estimar o preço à vista da commodity, com base nas informações dos preços futuros. Nesse modelo, o logaritmo do preço à vista é a soma desses $N$ fatores mais um termo de tendência. Cada um dos fatores evolui segundo um MRM. Os autores fazem uma aplicação para o caso de quatro fatores usando dados de preços diários de contratos futuros de petróleo, encontrando resultados que evidenciaram que todos os parâmetros de reversão são significativos.

Ainda, com base no modelo de Schwartz e Smith (2000), Aiube, Baidya e Tito (2008) propuseram uma extensão desse modelo, incorporando saltos nas variações de curto prazo para analisar os preços do petróleo. Segundo os autores, a dinâmica com saltos proposta nas variações de curto prazo restringe o uso do filtro de Kalman como metodologia de estimação, por esse motivo, aplicaram o filtro de partículas, mostrando que a inclusão dos saltos explica melhor a estrutura dos preços do petróleo.

0 modelo de dois fatores de Schwartz e Smith (2000) foi usado em outros diversos trabalhos mais recentes para o GN, como, por exemplo, em Cartea e Williams (2008), Pinto (2009), Guigues, Sagatizabal e Zubelli (2010), Hem et al. (2011) e Marotta (2011).

\subsection{O modelo de dois fatores de Schwartz e Smith}

No modelo de dois fatores de Schwartz e Smith (2000), os autores decompõem o logaritmo do preço à vista $\left(S_{t}\right)$ de uma commodity em dois fatores estocásticos não observáveis: um primeiro fator, $\left(\chi_{t}\right)$, relacionado às variações de curto prazo dos preços, e um segundo $\left(\xi_{t}\right)$, relacionado ao preço de equilíbrio de longo prazo. Tais fatores devem ser estimados a partir de observações dos preços nos mercados futuros, para depois ser determinado o preço à vista.

0 modelo considera que o fator $\chi_{t}$ evolui segundo o MRM de Ornstein-Uhlenbeck, sendo um caso particular onde $\bar{\chi}_{t}=0$. Ou seja, são choques transitórios e que revertem à média zero, tais como efeitos de variações temporárias de estoque e demanda e variações climáticas. Já o segundo fator, $\xi_{t}$, segue um MAB e representa os choques permanentes como mudanças estruturais nos custos de produção, expectativas de exaustão de reservas e inovações tecnológicas.

No modelo, os fatores estocásticos são definidos da seguinte forma:

$\ln \left(S_{t}\right)=\chi_{t}+\xi_{t}$

onde

$d \chi_{t}=-\kappa \chi_{t} d t+\sigma_{\chi} d z_{\chi}$

$d \xi_{t}=\mu_{\xi} d t+\sigma_{\xi} d z_{\xi}$

$d z_{\chi} d z_{\xi}=\rho_{\chi \xi} d t$

onde $\kappa$ é a velocidade de reversão à média de longo prazo, $\sigma_{\chi}$ é a volatilidade do fator $\chi_{t}, d z_{\chi}$ e $d z_{\xi}$ são incrementos do processo padrão de Wiener, $\mu_{\xi}$ é a tendência do fator $\xi_{t}$ e $\sigma_{\xi}$ é a volatilidade do fator $\xi_{t}$ . A correlação entre os dois fatores estocásticos é representada por $\rho_{\chi \xi}$.

Dado que os processos de $\chi_{t}$ e de $\xi_{t}$ têm distribuição normal (SCHWARTZ; SMITH, 2000), a expectância e a variância no tempo $t$ para variáveis com maturidade no tempo $T$ são dadas por:

$E\left[\ln \left(S_{T}\right) \mid S_{t}\right]=e^{-\kappa(T-t)} \chi_{t}+\xi_{t}+\mu_{\xi}(T-t)$

$\operatorname{Var}\left[\ln \left(S_{T}\right) \mid S_{t}\right]=\operatorname{Var}\left[\chi_{T} \mid \chi_{t}\right]+\operatorname{Var}\left[\xi_{T} \mid \xi_{t}\right]+2 \operatorname{Cov}\left[\left(\chi_{t}, \xi_{t}\right)\right]=\left(1-e^{-2 \kappa(T-t)}\right) \frac{\sigma_{\chi}^{2}}{2 \kappa}+\sigma_{\xi}^{2}(T-t)+2\left(1-e^{-\kappa(T-t)}\right) \frac{\sigma_{\chi} \sigma_{\xi} \rho_{\chi \xi}}{\kappa}$ 
e pela propriedade de log-normalidade:

$$
\begin{aligned}
& E\left[S_{T}\right]=\exp \left[E\left[\ln \left(S_{t}\right)\right]+\frac{1}{2} \operatorname{Var}\left[\ln \left(S_{t}\right)\right]\right]=\exp \left[e^{-\kappa(T-t)} \chi_{t}+\xi_{t}+\mu_{\xi}(T-t)+\right. \\
& \left.+\frac{1}{2}\left[\left(1-e^{-2 \kappa(T-t)}\right) \frac{\sigma_{\chi}^{2}}{2 \kappa}+\sigma_{\xi}^{2}(T-t)+2\left(1-e^{-\kappa(T-t)}\right) \frac{\sigma_{\chi} \sigma_{\xi} \rho_{\chi \xi}}{\kappa}\right]\right]
\end{aligned}
$$

0 modelo acima está escrito sob a medida real de probabilidade. A fim de valorar os contratos futuros de GN, é realizada a transformação para a medida neutra ao risco e procedida à derivação dos preços futuros sob o argumento de que não há arbitragem entre preços futuros e à vista. Essa derivação pode ser obtida seguindo as etapas descritas em Schwartz e Smith (2000).

Black e Scholes (1973) apresentaram a ideia de criar um portfólio e eliminar o risco do mesmo, fazendo com que a sua remuneração fosse idêntica à taxa livre de risco. Dessa forma, criou-se a condição de não arbitragem. Posteriormente, Harrison e Kreps (1979) e Harrison e Pliska (1981) trouxeram uma metodologia alternativa no apreçamento de derivativos, usando uma medida de probabilidade alternativa, e calcularam o valor esperado da opção segundo essa nova medida. Conforme a metodologia de Harrison e Kreps (1979) e Harrison e Pliska (1981), o Primeiro Teorema Fundamental de Finanças, considera que se o modelo de mercado possui uma medida de probabilidade neutra ao risco, então ele não admite arbitragem. Já o Segundo Teorema Fundamental de Finanças define que o mercado é completo se e somente se possui uma única medida neutra ao risco. Dessa forma, considerando o mercado completo, subtrai-se um prêmio de risco do processo inicialmente definido por Schwartz e Smith (2000).

Nas Equações 8 e 9 foram subtraídos os prêmios de risco ( $\lambda_{\chi}$ e $\lambda_{\xi}$ ), considerados constantes, referentes a ambos os fatores de incerteza das Equações 2 e 3, respectivamente:

$d \chi_{t}=\left(-\kappa \chi_{t}-\lambda_{\chi}\right) d t+\sigma_{\chi} d z_{\chi}^{*}$

$d \xi_{t}=\left(\mu_{\xi}-\lambda_{\xi}\right) d t+\sigma_{\xi} d z_{\xi}^{*}$

De acordo com Schwartz e Smith (2000), a Equação 10 mostra a expectância com a adaptação neutra ao risco:

$$
E\left[\ln \left(S_{T} \mid S_{t}\right)\right]=e^{-\kappa(T-t)} \chi_{t}-\frac{\lambda_{\chi}}{\kappa}\left(1-e^{-\kappa(T-t)}\right)+\xi_{t}+\left(\mu_{\xi}-\lambda_{\xi}\right)(T-t)
$$

Já a variância não sofre alteração, mantendo-se a mesma da Equação 6.

No processo neutro ao risco tem-se que $F_{t, T}=E\left[S_{T} \mid S_{t}\right]$, onde $F_{t, T}$ é o preço no tempo $t$ do contrato futuro com maturidade em $T(0 \leq t \leq T)$. De acordo com Schwartz e Smith (2000), utilizando a relação entre os momentos da distribuição log-normal e da normal, tem-se a Equação 11 deduzida por Schwartz e Smith (2000):

$$
\begin{aligned}
& \ln \left[F_{t, T}\right]=\ln \left[E\left(S_{T} \mid S_{t}\right)\right]=E\left[\ln \left(S_{T} \mid S_{t}\right)\right]+\frac{1}{2} \operatorname{Var}\left[\ln \left(S_{T} \mid S_{t}\right)\right]=e^{-\kappa(T-t)} \chi_{t}+\xi_{t}+\left(\mu_{\xi}-\lambda_{\xi}\right)(T-t)- \\
& \left(1-e^{-\kappa(T-t)}\right) \frac{\lambda_{\chi}}{\kappa}+\frac{1}{2}\left[\left(1-e^{-2 \kappa(T-t)}\right) \frac{\sigma_{\chi}^{2}}{2 \kappa}+\sigma_{\xi}^{2}(T-t)+2\left(1-e^{-\kappa(T-t)}\right) \frac{\rho_{\chi \xi} \sigma_{\chi} \sigma_{\xi}}{\kappa}\right]
\end{aligned}
$$

Considerando que o preço à vista das commodities como o GN não é diretamente observável, um conjunto de contratos futuros, técnicas de máxima verossimilhança e filtros de Kalman foram usados para projetar os preços, estimar os parâmetros do modelo e calibrá-lo. 0 modelo de Schwartz e Smith (2000) pode ser modelado de forma discreta através da abordagem de árvores binômias bivariáveis, que será detalhada na seção a seguir. 


\section{4. Árvore de eventos}

A árvore de eventos é uma das metodologias usadas no apreçamento de opções, devido a sua facilidade de implementação, versatilidade e precisão. Utilizada para modelos de tempo discreto, as árvores de eventos contêm nós de incerteza que descrevem os possiveis comportamentos dos fatores estocásticos. Nas árvores, a utilização de ramificações é flexível, podendo ser adaptada quando se altera a situação inicial. Essa flexibilidade torna a sua utilização atrativa para aplicações de modelagem de opções.

Cox, Ross e Rubinstein (1979) foram os precursores na avaliação de opções através de árvores de eventos, apresentando um modelo que generaliza o resultado de Black e Scholes (1973). Os autores propuseram a modelagem discreta por árvore binomial recombinante para avaliar opções cujo ativo-objeto segue um MGB. $\mathrm{Na}$ abordagem de Cox, Ross e Rubinstein (1979), os nós das ramificações se recombinam, o movimento de subida $(u)$ é o inverso do de descida $(d)$, fazendo com que em cada passo $N$ se obtenham $(N+1)$ nós e não $2 N$, caso não houvesse recombinação.

Boyle (1988) foi o primeiro a utilizar a abordagem de Cox, Ross e Rubinstein (1979) em um modelo trinomial para avaliação de opções com um ativoobjeto. Ao utilizar processos estocásticos de dois fatores, Boyle (1988) introduziu o conceito de árvore bivariável, que posteriormente foi seguido por Nelson e Ramaswamy (1990) em um método de sequência binomial, obtendo um modelo que pode ser utilizado para processos estocásticos que seguem tanto um MGB quanto um MRM discretos.

Um modelo discreto trinomial para um fator que segue o MRM foi construído por Hull e White (1994a) e usado por Jaillet, Ronn e Tompaidis (2004) para a previsão do preço do GN e cálculo de opções de swing. Como extensão do primeiro modelo, Hull e White (1994b) desenvolveram um modelo de árvore trinomial para dois fatores, ambos seguindo um MRM.

Hahn (2005) utilizou um modelo com dois fatores de incerteza para modelar o preço do petróleo e calcular uma opção de abandono de um poço de petróleo. Brandão, Hahn e Dyer (2005) também usaram árvores binomiais para valorar opções de investimento modelando o preço do petróleo como um MGB. Hahn e Dyer (2008) utilizaram essa mesma abordagem para modelar uma árvore bivariável combinando dois processos MRM, ambos segundo o modelo censurado de Nelson e Ramaswamy (1990).

Já Bastian-Pinto, Brandão e Hahn (2009), para avaliar uma opção de troca de input no setor sucroalcooleiro, desenvolveram uma árvore quadrinomial utilizando uma aproximação proposta por Nelson e Ramaswamy (1990) para um MRM. Já
Brandão e Dyer (2009) modelaram os riscos privado e de mercado de um projeto por meio de dois MGB correlacionados. Mais recentemente, Hahn e Dyer (2011) usaram dois fatores de risco e árvores binomiais bivariáveis para modelar em tempo discreto. Eles utilizaram um formato de árvores bidimensional para os mesmos problemas analisados por Schwartz e Smith (2000).

\section{1. Árvore binomial de Hahn e Dyer para dois fatores}

Hahn e Dyer (2011) desenvolveram um método para a construção de uma árvore binomial bivariável aplicável ao modelo de dois fatores de Schwartz e Smith (2000). 0 primeiro passo consiste em modelar duas árvores binomiais, uma para cada um dos dois fatores estocásticos de risco, isto é, para $\xi_{t}$ e $\chi_{t}$. Esse modelo discreto é uma sequência de dois nós. Um nó binomial para o MAB da variável de longo prazo, $\xi$, seguido de nós binomiais condicional para o MRM da variável de curto prazo, $\chi$. A solução é representar uma árvore binomial bivariável como mostra a Figura 1.

A árvore resultante pode ser representada como o produto de um processo binomial para $\xi$ e um processo binomial condicional para $\chi$. Na árvore obtida cada nó terá quatro ramificações, devido à combinação dos ramos das árvores de $\xi_{t}$ e $\chi_{t}$, como pode ser observado na Figura 2.

Para definir as probabilidades conjuntas para cada um dos nós, Hahn e Dyer (2011) assumem que os incrementos $(\Delta \xi e \Delta \chi)$ e as tendências $\left(v_{\xi} e v_{\chi}\right)$ para cada um dos fatores de risco são especificados da seguinte forma:

$$
\Delta \xi=\sigma_{\xi} \sqrt{\Delta t} ; \quad \Delta \chi=\sigma_{\chi} \sqrt{\Delta t} ; \quad v_{\xi}=\mu_{\xi} ; \quad v_{\chi}=-\kappa \chi_{t}
$$

As probabilidades conjuntas representam as quatro possíveis combinações de subida e descida dos dois fatores. Usando o mesmo método empregado por Boyle (1988), as probabilidades para os quatro nós resultantes são obtidas com a combinação das médias e variâncias dos dois fatores estocásticos, conforme demonstrado por Hahn e Dyer (2008; 2011). Pode ser necessário induzir um determinado desvio para os resultados de longo prazo nos nós onde as probabilidades sejam maiores que um ou menores que zero. Caso a probabilidade calculada seja maior do que um, ajusta-se acrescentando um desvio negativo de forma que essa probabilidade passe a ser um. Segundo Hahn e Dyer (2011), a implementação desse modelo discreto é mais eficiente aplicando recombinações bidimensionais. Aplica-se a regra de Bayes para decompor as probabilidades 

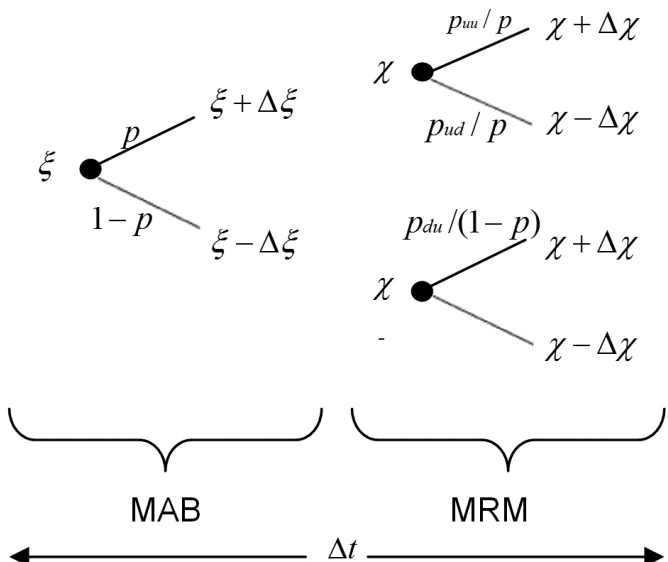

Figura 1. Ramificações da árvore binomial de dois fatores.

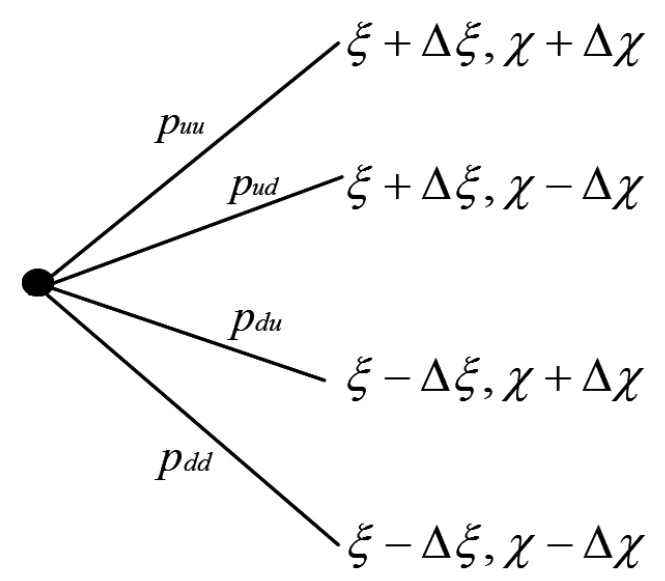

Figura 2. Ramificações da árvore binomial de dois fatores.

conjuntas no produto das marginais e condicionais, ou seja, $p\left(\chi_{t}, \xi_{t}\right)=p\left(\chi_{t} \mid \xi_{t}\right) p\left(\xi_{t}\right)$. Assim, as probabilidades marginais de subida e descida, em nível de equilíbrio, são, respectivamente:

$\left\{\begin{array}{l}p_{u}=\frac{1}{2}+\frac{1}{2} \frac{v_{\xi} \Delta t}{\Delta \xi} \\ p_{d}=1-p_{u}\end{array}\right.$

e as probabilidades condicionais são:

$$
\left\{\begin{array}{l}
p_{u \mid u}=\frac{\Delta \xi\left(\Delta \chi+\Delta t v_{\chi}\right)+\Delta t\left(\Delta \chi v_{\xi}+\rho \sigma_{\chi} \sigma_{\xi}\right)}{2 \Delta \chi\left(\Delta \xi+\Delta t v_{\xi}\right)} \\
p_{d \mid u}=\frac{\Delta \xi\left(\Delta \chi-\Delta t v_{\chi}\right)+\Delta t\left(\Delta \chi v_{\xi}-\rho \sigma_{\chi} \sigma_{\xi}\right)}{2 \Delta \chi\left(\Delta \xi+\Delta t v_{\xi}\right)} \\
p_{u \mid d}=\frac{\Delta \xi\left(\Delta \chi-\Delta t v_{\chi}\right)+\Delta t\left(\rho \sigma_{\chi} \sigma_{\xi}-\Delta \chi v_{\xi}\right)}{2 \Delta \chi\left(\Delta \xi+\Delta t v_{\xi}\right)} \\
p_{d \mid d}=\frac{\Delta \xi\left(\Delta \chi+\Delta t v_{\chi}\right)-\Delta t\left(\Delta \chi v_{\xi}+\rho \sigma_{\chi} \sigma_{\xi}\right)}{2 \Delta \chi\left(\Delta \xi+\Delta t v_{\xi}\right)}
\end{array}\right.
$$

Como o processo é neutro ao risco, onde não há a possibilidade de arbitragem, pode-se fazer o desconto através da taxa livre de risco, considerando os prêmios de risco $\lambda_{\chi}$ e $\lambda_{\xi}$ para cada um dos fatores. Para isso, os prêmios de risco têm que penalizar as tendências dos dois fatores de incerteza da seguinte forma:

$$
v_{\chi}^{*}=-\kappa \chi_{t}-\lambda_{\chi} \quad \text { e } \quad v_{\xi}^{*}=\mu_{\xi}-\lambda_{\xi}
$$

Sendo assim, de acordo com Pinto (2009), as probabilidades marginais, na forma neutra ao risco, são:

$\left\{\begin{array}{l}p_{u}=\frac{1}{2}+\frac{1}{2} \frac{\left(\mu_{\xi}-\lambda_{\xi}\right) \sqrt{\Delta t}}{\sigma_{\xi}}=\frac{1}{2}+\frac{1}{2} \frac{v_{\xi}^{*} \Delta t}{\Delta \xi} \\ p_{d}=1-p_{u}=\frac{1}{2}-\frac{1}{2} \frac{\left(\mu_{\xi}-\lambda_{\xi}\right) \sqrt{\Delta t}}{\sigma_{\xi}}\end{array}\right.$

E as probabilidades condicionais podem ser reescritas da seguinte forma:

$$
\left\{\begin{array}{l}
p_{u \mid u}=\frac{\Delta \xi\left(\Delta \chi+\Delta t v_{\chi}^{*}\right)+\Delta t\left(\Delta \chi v_{\xi}^{*}+\rho \sigma_{\chi} \sigma_{\xi}\right)}{2 \Delta \chi\left(\Delta \xi+\Delta t v_{\xi}^{*}\right)} \\
p_{d \mid u}=\frac{\Delta \xi\left(\Delta \chi-\Delta t v_{\chi}^{*}\right)+\Delta t\left(\Delta \chi v_{\xi}^{*}-\rho \sigma_{\chi} \sigma_{\xi}\right)}{2 \Delta \chi\left(\Delta \xi+\Delta t v_{\xi}^{*}\right)} \\
p_{u \mid d}=\frac{\Delta \xi\left(\Delta \chi-\Delta t v_{\chi}^{*}\right)+\Delta t\left(\rho \sigma_{\chi} \sigma_{\xi}-\Delta \chi v_{\xi}^{*}\right)}{2 \Delta \chi\left(\Delta \xi+\Delta t v_{\xi}^{*}\right)} \\
p_{d \mid d}=\frac{\Delta \xi\left(\Delta \chi+\Delta t v_{\chi}^{*}\right)-\Delta t\left(\Delta \chi v_{\xi}^{*}+\rho \sigma_{\chi} \sigma_{\xi}\right)}{2 \Delta \chi\left(\Delta \xi+\Delta t v_{\xi}^{*}\right)}
\end{array}\right.
$$

Hahn e Dyer (2011) aplicaram esse modelo discreto para o mesmo exemplo discutido por Schwartz e Smith (2000). No presente trabalho será aplicado esse modelo para o preço do GN e avaliação de opções de swing em contratos de GN. Já com a árvore binominal bivariável dos preços à vista do GN para o período de validação do contrato, pode-se calcular o valor da flexibilidade embutida no contrato através da opção de swing cujas características e especificações são definidas na próxima seção.

\section{A opção de swing}

Uma das maiores dificuldades no mercado de opções é determinar o valor do prêmio, uma vez que uma estimação malfeita pode levar à perda de rentabilidade tanto para o lançador quanto para o comprador. Os modelos mais tradicionais de cálculo de prêmio de opções são o modelo de Black e Scholes (1973), que considera tempo contínuo, e o modelo binomial que é um modelo em tempo discreto.

São várias as opções exóticas encontradas no mercado, cada qual com as suas especificações. 
As opções de swing são definidas como o direito de comprar, em momentos específicos, volumes flexíveis a certos preços pré-fixados. De modo geral, um contrato de swing vem associado a um contrato padrão que garante ao seu detentor o fornecimento de um determinado montante da mercadoria, por um período de tempo determinado e com um preço predeterminado. Esse contrato é composto de uma parte fixa e uma variável. A parte fixa permite que o detentor compre, em um período especificado $\left[T_{1}\right.$, $T_{2}$ ], $0 \leq T_{1} \leq T_{2}$, certo volume básico, por um preço predeterminado. A parte swing (variável) do contrato permite flexibilidade ao redor do volume contratado no montante de entrega. Permitindo ao detentor da opção exercer $N$ direitos que podem ter significados diferentes de acordo com o contrato. 0 direito pode ser exercido apenas em determinadas datas $\left\{\tau_{1}, \ldots, \tau_{2}\right\}$, onde $T_{1} \leq \tau_{1}<\tau_{2}<\ldots<\tau_{n} \leq T_{2}$, e somente uma vez em cada data. Além disso, se um direito é exercido, existe um tempo de refração $\Delta t_{R}$, o que limita a próxima vez que um direito pode ser novamente exercido. Se o tempo entre o exercício da opção e a entrega efetiva da carga for maior que o período entre dois possíveis exercícios, a restrição de refração deve ser incluída no contrato, caso contrário essa refração se torna redundante, onde $\Delta t_{R} \leq \min _{1 \leq j \leq n-1}\left(\tau_{j+1}-\tau_{j}\right)$.

As duas principais categorias de contratos dependem da duração do efeito associado com o exercício de um direito são:

- Efeito local - o exercício de um direito modifica o volume de entrega somente na data do exercício e a entrega será revertida para o nível do volume contratado;

- Efeito global - o exercício de um direito modifica o volume de entrega para as próximas datas e a entrega continua com o novo volume até o próximo exercício.

\subsection{Opções de swing nos contratos de gás natural}

Um contrato de longo prazo de GN pode ser visto como um conjunto de contratos futuros, conforme a flexibilidade de cada um. Geralmente, os contratos de GN incorporam cláusulas de pagamento por volume mínimo, mesmo que este não seja utilizado, conhecidas como cláusulas take-or-pay, que podem ser consideradas como um mecanismo de repasse do custo dos investimentos específicos realizados ao longo da cadeia até o consumidor final. Essas cláusulas funcionam como uma penalidade a ser aplicada ao comprador pela quebra contratual ou diminuição do consumo.

No caso brasileiro, normalmente, o comprador de GN tem o direito de não adquirir parte do volume contratado, isto é, o take-or-pay é inferior a 100\%, mas tem garantia do fornecimento de todo o volume contratado. 0 contrato pode se assemelhar a um conjunto de opções de venda (caso consuma menos do que o volume contratado) e de compra (caso consuma mais do que o volume contratado), para cada período de fornecimento dentro do prazo contratual, por causa da flexibilidade entre a capacidade contratada e o volume consumido. No estudo de caso da próxima seção, o contrato analisado leva em consideração somente as opções de compra existentes.

Dado que no Brasil os contratos são específicos, confidenciais e personalizados para cada cliente, sendo documentos extremamente restritos dentro da empresa, neste trabalho optou-se por analisar um contrato com as mesmas características do usado por Jaillet, Ronn e Tompaidis (2004), para avaliar o impacto das flexibilidades contratuais na indústria brasileira de GN. Nesse contrato com opção de swing, a cada direito o titular do contrato escolhe por um volume incremental positivo, ou simplesmente não exerce esse direito. 0 volume incremental tem limitações físicas que restringem o volume total a um mínimo e máximo a serem contratados. 0 volume total entregue no período também tem restrições especificadas no contrato, cuja violação pode ser permitida, mas há penalidades. É necessário especificar também um preço de exercício da opção. 0 procedimento de análise e valoração de um contrato começa na data de expiração da opção, e é feito através de indução retroativa, do vencimento da opção até o instante presente. Em cada data, a possibilidade de exercício é considerada, tendo o valor da opção como o máximo entre não exercer um direito de swing, ou exercer e mudar para um novo processo igual, mas com um menor número de direitos de exercício.

\section{Estudo de caso}

No estudo de caso, o modelo de Schwartz e Smith (2000) com incorporação de sazonalidade trimestral é utilizado para modelar e projetar os preços do GN, e no contexto binomial baseado em Hahn e Dyer (2011) são valoradas as opções de swing. 0 contrato considerado embute uma opção de swing com quatro datas de exercício, em que só podem ser exercidos no máximo dois direitos. 0 tempo de refração não será levado em consideração e o contrato faz parte da categoria cujo efeito é local, ou seja, afeta o volume de entrega somente na data do exercício. Em cada exercício, o titular do contrato de swing pode escolher um consumo positivo extra de um ou dois MMBtus de GN, ou pode não exercer esse direito e consumir somente o GN que foi inicialmente contratado. 0 exercício ocorre no último dia do mês em que o contrato do mês seguinte é negociado. É considerado um horizonte de tempo de um ano, com possíveis 
exercícios trimestrais. Há duas possíveis estruturas de preços de exercício:

1a) Preço de exercício fixado em US $\$ 3,70$ por MMBtu, média dos últimos 12 meses do contrato futuro para quatro meses à frente;

$2^{\text {a) }}$ Preço de exercício variável baseado na estrutura dos preços futuros para as diversas datas de exercício.

No estudo de caso, só será analisada a parte variável do contrato, sem considerar multas e penalidades. E demais custos burocráticos, fiscais e operacionais são desconsiderados nos cálculos.

Como o processo é neutro ao risco, o desconto é feito através da taxa livre de risco, considerando os prêmios de risco $\lambda_{\chi}$ e $\lambda_{\xi}$ para cada um dos fatores. Significa que não há possibilidade de arbitragem. A taxa de juros livre de risco é considerada constante e igual a 5\% ao ano, uma aproximação da taxa livre de risco deflacionada brasileira em maio de 2012. De acordo com ata do Banco Central do Brasil (2012), a taxa de juros nominal de referência (Selic) em maio de 2012 era de 9\% ao ano e a taxa de inflação (IGP-M) de 12 meses era de 4,26\% ao ano.

As características do contrato de GN usado no estudo de caso podem ser resumidas da seguinte forma:

- Horizonte no tempo: 1 ano com exercícios trimestrais;

- Data da assinatura do contrato: 2 de maio de 2012;

- Preço de exercício: fixo ou variável (análise dos dois casos);

- Número máximo de exercícios: 2;

- Processo estocástico para os preços do GN: modelo de dois fatores de Schwartz e Smith (2000) com sazonalidade trimestral;

- Taxa de juros livre de risco: $r=5 \%$ aa;

- Ausência de multas, penalidades, impostos e demais custos.

\subsection{Análise da série de preços do gás natural e estimação dos parâmetros do modelo}

Em geral, as commodities não são negociadas nos mercados à vista fazendo com que os preços à vista não sejam diretamente observáveis. Sob o ponto de vista acadêmico, segundo Aiube (2013), é comum usar os contratos futuros com vencimento mais próximo como uma proxy para o preço à vista. Neste trabalho, os dados básicos utilizados para análise dos modelos foram os preços da commodity GN Henry $H u b$, negociada na Bolsa de Mercadorias de Nova York (Nymex). Os dados utilizados correspondem aos valores coletados do banco de dados do serviço Bloomberg, diariamente, e, quando semanalmente, do fechamento da quarta-feira, no período de janeiro de 2000 a abril de 2012. No caso de feriado, o valor de quinta-feira é o apurado. Foram analisados contratos para diversos vencimentos, como resumem a Tabela 1 e a Figura 3.

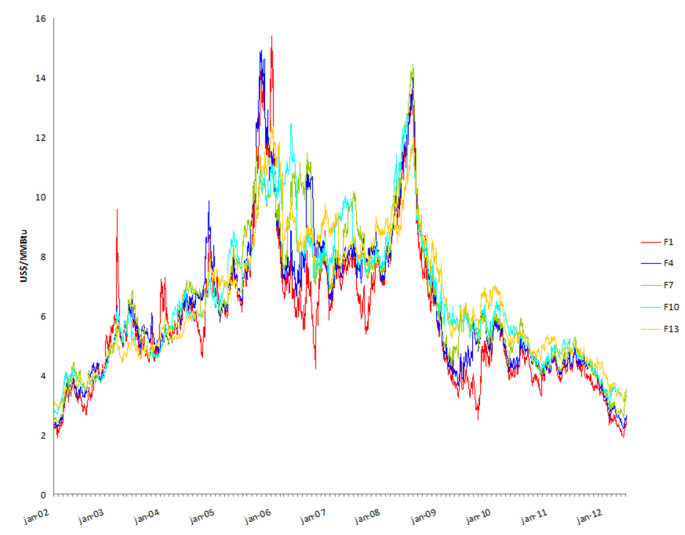

Figura 3. Preços futuros do gás natural - Henry Hub.

Tabela 1. Informações das séries de preços futuros do GN utilizadas.

\begin{tabular}{|c|c|c|c|c|c|}
\hline Período & $\begin{array}{l}\text { Número de } \\
\text { dados }\end{array}$ & Contrato & $\begin{array}{c}\text { Média } \\
\text { US\$/MMBtu }\end{array}$ & $\begin{array}{c}\text { Desvio padrão } \\
\text { US\$/MMBtu }\end{array}$ & $\begin{array}{c}\text { Média } 12 \text { meses } \\
\text { US\$/MMBtu }\end{array}$ \\
\hline \multirow{5}{*}{$\begin{array}{c}5 / 1 / 2000 \\
a \\
28 / 3 / 2012\end{array}$} & \multirow{5}{*}{$\begin{array}{c}762 \\
\text { semanais }\end{array}$} & F1 & 5,65 & 2,37 & 3,69 \\
\hline & & $\mathrm{F} 4$ & 5,95 & 2,41 & 3,90 \\
\hline & & F7 & 6,08 & 2,38 & 4,06 \\
\hline & & F10 & 6,12 & 2,29 & 4,21 \\
\hline & & F13 & 6,10 & 2,23 & 4,32 \\
\hline \multirow{5}{*}{$\begin{array}{c}4 / 1 / 2002 \\
a \\
30 / 4 / 2012\end{array}$} & \multirow{5}{*}{$\begin{array}{c}2.589 \\
\text { diários }\end{array}$} & $\mathrm{F} 1$ & 5,85 & 2,37 & 3,43 \\
\hline & & $\mathrm{F} 4$ & 6,26 & 2,42 & 3,68 \\
\hline & & F7 & 6,44 & 2,36 & 3,86 \\
\hline & & F10 & 6,52 & 2,24 & 4,03 \\
\hline & & F13 & 6,52 & 2,14 & 4,19 \\
\hline \multirow{5}{*}{$\begin{array}{c}2 / 1 / 2009 \\
a \\
30 / 4 / 2012\end{array}$} & \multirow{5}{*}{$\begin{array}{l}837 \\
\text { diários }\end{array}$} & $\mathrm{F} 1$ & 4,01 & 0,79 & 3,43 \\
\hline & & $\mathrm{F} 4$ & 4,40 & 0,79 & 3,68 \\
\hline & & F7 & 4,72 & 0,87 & 3,86 \\
\hline & & F10 & 5,00 & 0,88 & 4,03 \\
\hline & & F13 & 5,19 & 0,94 & 4,19 \\
\hline
\end{tabular}


Com base no modelo de Schwartz e Smith (2000), onde o logaritmo do preço futuro do GN é linear com relação aos dois fatores de risco, e incorporando uma componente determinística sazonal, os preços foram estimados usando filtros de Kalman. Dessa maneira, os hiperparâmetros do modelo e os fatores de risco $\xi_{t}$ e $\chi_{t}$ podem ser estimados por meio da maximização da verossimilhança do erro de previsão. Os filtros de Kalman e os procedimentos foram implementados com o uso do software EViews, da mesma forma que Aiube (2013), introduzindo o componente sazonal determinístico, $f(t)$. 0 componente sazonal foi implementado usando variáveis dummies trimestrais, a fim de mensurar os impactos que as diferentes estações do ano exercem sobre os preços do GN. Marotta (2011) calculou os valores dos hiperparâmetros com base nas séries de preços futuros do GN para o modelo de Schwartz e Smith (2000). Esses dados foram usados como valores iniciais na estimação.

0 filtro de Kalman é um procedimento recursivo eficiente de estimação para calcular estimativas de variáveis de estado (no contexto de Kalman são variáveis não observáveis) através de variáveis observáveis. É um procedimento que determina o estimador ótimo, dadas as informações disponíveis até o tempo $t$. Esse estimador é dito ótimo uma vez que a variância do erro das variáveis de estado é mínima. As equações do filtro de Kalman são agrupadas em dois tipos: as equações de transição que regem as variáveis de estado e as equações de medição que relacionam as variáveis de estado e os preços observados. As equações de transição são responsáveis pelo avanço das variáveis de estado e das covariâncias para se obter as primeiras estimativas para o próximo instante de tempo. Já as equações de medição incorporam uma nova informação da variável observável nas estimativas anteriores para obter melhores estimadores.

Com base na Equação 11 dos preços futuros do modelo de Schwartz e Smith (2000), acrescentando a função de sazonalidade, $f(t)$,

$\ln \left(F_{t, T}\right)=e^{-\kappa(T-t)} \chi_{t}+\xi_{t}+A(T-t)+f(t)+u_{t}$

Onde $F_{\mathrm{t}, \mathrm{T}}$ é o preço futuro em $t$ de um contrato com vencimento em $T, u_{\mathrm{t}}$ é um ruído gaussiano, $u_{\mathrm{t}} \sim \mathrm{N}\left(0, s_{\mathrm{i}}\right), A(T-t)$ é dado por:

$A(T-t)=\left(\mu_{\xi}-\lambda_{\xi}\right)(T-t)-\left(1-e^{-\kappa(T-t)}\right) \frac{\lambda_{\chi}}{\kappa}+\frac{1}{2}\left[\left(1-e^{-2 \kappa(T-t)}\right) \frac{\sigma_{\chi}^{2}}{2 \kappa}+\sigma_{\xi}^{2}(T-t)+2\left(1-e^{-\kappa(T-t)}\right) \frac{\rho_{\chi \xi} \sigma_{\chi} \sigma_{\xi}}{\kappa}\right]$

E a função de sazonalidade $f(t)$ é representada pela Equação 17:

$f(t)=\alpha_{2} Q_{2}+\alpha_{3} Q_{3}+\alpha_{4} Q_{4}$

onde $\alpha_{2}, \alpha_{3}$ e $\alpha_{4}$ representam os coeficientes do segundo trimestre, terceiro trimestre e quarto trimestre, respectivamente. As variáveis $Q_{2}, Q_{3}$ e $Q_{4}$ são variáveis dummies e referem-se aos respectivos trimestres, tendo o $1^{\circ}$ trimestre como padrão.

Os parâmetros a serem estimados, através da maxi-mização da verossimilhança do erro de previsão, são: $\Theta=\left(\kappa, \lambda_{\chi}, \sigma_{\chi}, \mu_{\xi}, \lambda_{\xi}, \sigma_{\xi}, \rho_{\chi \xi}, s_{1}, s_{4}, s_{7}, s_{10}, s_{13}, \alpha_{2}, \alpha_{3}, \alpha_{4}\right)$, onde $s_{\mathrm{i}}$ é o desvio-padrão de ruído das observações do i-ésimo contrato.

Assim, no modelo de Schwartz e Smith (2000) as Equações 2 e 3 das variáveis de estado devem ser discretizadas. Após a discretização, obtêm-se as equações denominadas equações de transição:

$$
\begin{aligned}
\chi_{t} & =(1-\kappa \Delta t) \chi_{t-\Delta t}+\varepsilon_{t} \\
\xi_{t} & =\xi_{t-\Delta t}+\mu_{\xi} \Delta t+v_{t}
\end{aligned}
$$

onde $\varepsilon_{t} \sim N\left(0, \sigma_{\varepsilon}^{2}\right)$ e $v_{t} \sim N\left(0, \sigma_{v}^{2}\right)$, sendo $\rho_{\chi \xi} \Delta t$ a correlação entre as duas distribuições normais e $\Delta t \quad 0$ intervalo de tempo. Como as distribuições das variáveis $\xi_{t}$ e $\chi_{t}$ são condicionalmente normais, as variâncias condicionais e a matriz de covariância são dadas por:

$$
\operatorname{Var}\left[\chi_{t} \mid \chi_{t-1}\right]=\left(1-e^{-2 \kappa(\Delta t)}\right) \frac{\sigma_{\chi}^{2}}{2 \kappa}=\sigma_{\varepsilon}^{2} \operatorname{Var}\left[\xi_{t} \mid \xi_{t-1}\right]=\sigma_{\xi}^{2} \Delta t=\sigma_{v}^{2} \operatorname{Cov}\left[\chi_{t}, \xi_{t}\right]=\left[\begin{array}{cc}
\left(1-e^{-2 \kappa(\Delta t)}\right) \frac{\sigma_{\chi}^{2}}{2 \kappa} & \left(1-e^{-\kappa(\Delta t)}\right) \frac{\sigma_{\chi} \sigma_{\xi} \rho_{\chi_{\xi}}}{\kappa} \\
\left(1-e^{-\kappa(\Delta t)}\right) \frac{\sigma_{\chi} \sigma_{\xi} \rho_{\chi \xi}}{\kappa} & \sigma_{\xi}^{2}(\Delta t)
\end{array}\right]
$$


Já tendo as equações de transição, a equação de medição é dada pela Equação 16 , onde $u_{t} \sim N\left(0, \sigma_{u}^{2}\right)$ é um ruído gaussiano que foi introduzido no logaritmo dos preços futuros, cujos erros são descorrelacionados dos erros das equações de transição (18) e (19).

De posse de todas as equações e ruídos, é possivel implementar o algoritmo do filtro de Kalman e, a cada rodada, atualizar a estimativa para os fatores não observáveis. Para minimizar o erro quadrático das previsões, é preciso encontrar o estimador ótimo através da maximização da função verossimilhança, dado o vetor inicial de hiperparâmetros. A Tabela 2 mostra os resultados da estimação, após 10 mil interações.

Com base em dados dos preços diários no período de 2002 a 2012, foram calculadas as estimativas dos parâmetros que serviram de base na projeção dos preços do GN para datas de exercício da opção.

Os estimadores, em sua maioria, tanto para os dados diários quanto para os dados semanais, são bastante similares, porém a sazonalidade é mais significativa na análise dos dados diários. Observa-se que o prêmio de risco do fator de longo prazo, $\lambda_{\xi}$ tem sinal negativo para todas as séries parametrizadas, o que parece um contrassenso. No entanto, em Marotta (2011) e Schwartz e Smith (2000), o resultado encontrado também foi negativo para o prêmio de risco de longo prazo, impactando de forma positiva na taxa de crescimento neutra ao risco $\left(\mu_{\xi}-\lambda_{\xi}\right)$. Já a velocidade de reversão à média de longo prazo $\kappa$ apresenta um nível alto de modo semelhante aos estudos anteriores analisados como Marotta (2011) e Manoliu e Tompaidis (2000) para o preço do GN. A correlação entre os dois fatores estocásticos, $\rho_{\chi \xi}$ é alta para todas as séries parametrizadas, assim como em Manoliu e Tompaidis (2000) para os preços do GN e em Schwartz e Smith (2000) para o preço do petróleo.

Com base nas estimativas encontradas, os parâmetros para os preços diários com dados de 2002 a 2012 se mostraram mais consistentes e serviram de base para a previsão dos preços do GN.

De acordo com os cálculos efetuados, os valores iniciais das duas variáveis ou fatores de risco para a data de assinatura do contrato (maio de 2012) são os seguintes: $\chi_{0}=-0,3097$ e $\xi_{0}=1,1012$.

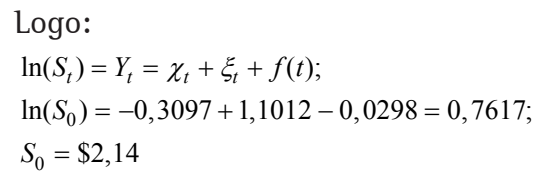

Tabela 2. Parâmetros estimados.

\begin{tabular}{|c|c|c|c|c|c|c|}
\hline \multirow{2}{*}{ Parâmetro } & \multicolumn{2}{|c|}{ Dados semanais 2000-2012 } & \multicolumn{2}{|c|}{ Dados diários 2002-2012 } & \multicolumn{2}{|c|}{ Dados diários 2009-2012 } \\
\hline & Valor estimado & Erro & Valor estimado & Erro & Valor estimado & Erro \\
\hline$\mu_{\xi}$ & 0,0093 & 0,0408 & $-0,3612$ & 0,0650 & $-0,0886$ & 0,1103 \\
\hline$\lambda_{\xi}$ & $-0,1757$ & 0,0407 & $-0,6032$ & 0,0648 & $-0,1873$ & 0,1116 \\
\hline$\kappa$ & 3,1389 & 0,0176 & 3,2919 & 0,0072 & 2,2055 & 0,0772 \\
\hline$\lambda_{\chi}$ & 0,3220 & 0,0376 & 0,5887 & 0,0197 & 0,0637 & 0,0222 \\
\hline$\sigma_{\chi}$ & 0,4814 & 0,0104 & 0,4816 & 0,0067 & 0,3052 & 0,0083 \\
\hline$\sigma_{\xi}$ & 0,1860 & 0,0055 & 0,2181 & 0,0039 & 0,2410 & 0,0063 \\
\hline$\rho_{\chi \xi}$ & 0,5507 & 0,0220 & 0,6646 & 0,0086 & 0,6473 & 0,0233 \\
\hline$S_{1}$ & $4,68 \mathrm{E}-07$ & 1,85E-05 & 2,49E-07 & $8,56 \mathrm{E}-06$ & 0,0002 & $1,10 \mathrm{E}-05$ \\
\hline$S_{4}$ & 0,0012 & 1,07E-05 & 0,0009 & $3,29 \mathrm{E}-06$ & 0,0051 & 0,0003 \\
\hline$S_{7}$ & 0,0011 & 1,45E-05 & 0,0009 & 4,71E-06 & 0,0054 & 0,0004 \\
\hline$S_{10}$ & 0,0041 & 0,0001 & 0,0024 & 3,07E-05 & 0,0040 & 0,0003 \\
\hline$S_{13}$ & 0,0005 & $1,38 \mathrm{E}-05$ & 0,0005 & 4,79E-06 & 1,17E-08 & 3,57E-06 \\
\hline$\alpha_{2}$ & 0,0019 & 0,0070 & $-0,0298$ & 0,0064 & $-0,0197$ & 0,0128 \\
\hline$\alpha_{3}$ & 0,0111 & 0,0086 & 0,0141 & 0,0068 & $-0,0181$ & 0,0121 \\
\hline$\alpha_{4}$ & 0,0018 & 0,0080 & 0,0136 & 0,0067 & $-0,0111$ & 0,0094 \\
\hline
\end{tabular}


Além disso:

$$
\begin{aligned}
& \Delta \chi=\sigma_{\chi} \sqrt{\Delta t}=0,4816 \sqrt{\frac{1}{4}}=0,2408 ; \quad \Delta \xi=\sigma_{\xi} \sqrt{\Delta t}=0,2181 \sqrt{\frac{1}{4}}=0,1091 \\
& v_{\chi}^{*}=-\kappa \chi_{t}-\lambda_{\chi}=-3,2919 \chi_{t}-0,5887 ; \quad v_{\xi}^{*}=\mu_{\xi}-\lambda_{\xi}=-0,3612+0,6032=0,2420
\end{aligned}
$$

\subsection{Cálculo da opção de swing no contrato de gás natural}

0 valor da opção será determinado para duas estruturas de preços de exercício: na primeira o preço de exercício é fixado em US $\$ 3,70$ por MMBtu, média dos últimos 12 meses do contrato futuro para quatro meses à frente. Na segunda o preço de exercício é variável e está baseado na estrutura dos preços futuros para as diversas datas de exercício.

Caso 1: preço de exercício da opção fixado em US\$3,70 por MMBtu

Com a previsão dos preços já efetuada, foram calculados os valores das opções de swing seguindo a abordagem de Jaillet, Ronn e Tompaidis (2004), através da construção de três árvores binomiais bivariáveis: uma primeira quando nenhum direito é exercido, uma segunda quando unicamente um direito é exercido, e uma terceira quando os dois direitos são exercidos. Como essa árvore é bastante extensa, a Figura 2 contém parte da árvore com as quatro ramificações para cada instante de tempo e mostra os valores da opção de swing.

As três árvores podem ser explicadas da seguinte forma, lembrando que a análise é feita através de indução retroativa, do vencimento da opção até o instante presente e o preço de exercício é de US\$3,70 por MMBtu:

- 0 nível inferior mostra o valor da opção sem exercícios realizados. Os zeros na Figura 2 são atribuídos como consequência da ausência de penalidades e multas;

- 0 nível médio mostra a árvore quando um direito é exercido e dois MMBtus de GN extras são comprados. Nessa árvore, o valor para o primeiro nó do retângulo superior no instante $T 3$ é dado por:

$$
\begin{aligned}
& \max \left[2\left(P_{G N_{-} T 3}-P_{\text {Exrcicio }}\right)+0 ; \mathrm{P}_{o p \varsigma \tilde{a} o 1_{-} T 4} e^{-r \Delta t}\right] \\
& \max \left[2(5,0574-3,70)+0 ;\left(6,53 p_{u u}+1,2059 p_{u d}+3,8004 p_{d u}+0,00 p_{d d}\right) e^{-r \Delta t}\right] \\
& \max \left[2(1,3574)+0 ; \quad(1,2006) e^{-0,05 / 4}\right] \\
& \max [2,7148 ; 1,1857]=2,7148
\end{aligned}
$$

0 resultado mostra que é ideal exercer a opção de swing nesse nó para o máximo possível de MMBtus ao invés de esperar, pois o ganho com a diferença entre o preço à vista do GN e o preço de exercício é maior do que o valor da espera (os valores da opção em $T 4$ ponderados, descontados à taxa livre de risco para o período T3). Ao exercer essa opção, não é possível permanecer nessa árvore e, consequentemente, pula-se para a árvore inferior onde não é possível exercer nenhuma opção, seguindo o caminho indicado na Figura 4. No nível mais alto da árvore, o valor da opção de swing com dois direitos de exercício em cada nó é dado pelo maior valor entre: o valor de exercer um direito nesse nó somado ao valor presente da opção embutida na árvore situada abaixo (no nível médio, onde se tem um direito de exercício), ou o valor de se adiar o exercício.

Nessa árvore, o valor de US\$3,90 no primeiro nó do retângulo superior para o instante de tempo T3 é obtido por:

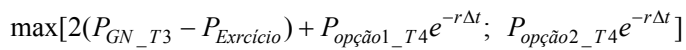

$$
\begin{aligned}
& \max \left[2(5,0574-3,70)+\left(6,53 p_{u u}+1,2059 p_{u d}+3,8004 p_{d u}+0,00 p_{d d}\right) e^{-r \Delta t} ;\right. \\
& \left.\left(6,53 p_{u u}+1,2059 p_{u d}+3,8004 p_{d u}+0,00 p_{d d}\right) e^{-r \Delta t}\right] \\
& \max \left[2(1,3574)+(1,2006) e^{-0,05 / 4} ;(1,2006) e^{-0,05 / 4}\right] \\
& \max [3,9005 ; 1,1857]=3,9005
\end{aligned}
$$

Nesse nó, o exercício da opção de swing é mais valioso do que a espera, portanto, é válido exercer a opção. Ao exercer essa opção, pula-se para a árvore do nível médio, onde ainda é possível exercer outra opção, seguindo o caminho indicado na Figura 4. 


\section{Caso 2: preço de exercício variável}

Nesse caso o preço de exercício da opção é diferente para cada uma das datas de exercício. Observando os contratos futuros do Henry $\mathrm{Hub}$ negociados no Nymex, na data de assinatura do contrato fixam-se os preços de exercícios pelos preços futuros para os meses de possíveis exercícios. 0 fechamento dos contratos futuros para os meses de interesse estão na Tabela 3.

Os cálculos para a opção de swing com diferentes preços de exercício foram feitos da mesma forma que os explicados anteriormente para o caso 1. A Figura 5 mostra o mesmo esquema da Figura 4, com a diferença de que o preço de exercício varia para cada uma das datas de possível exercício da opção.

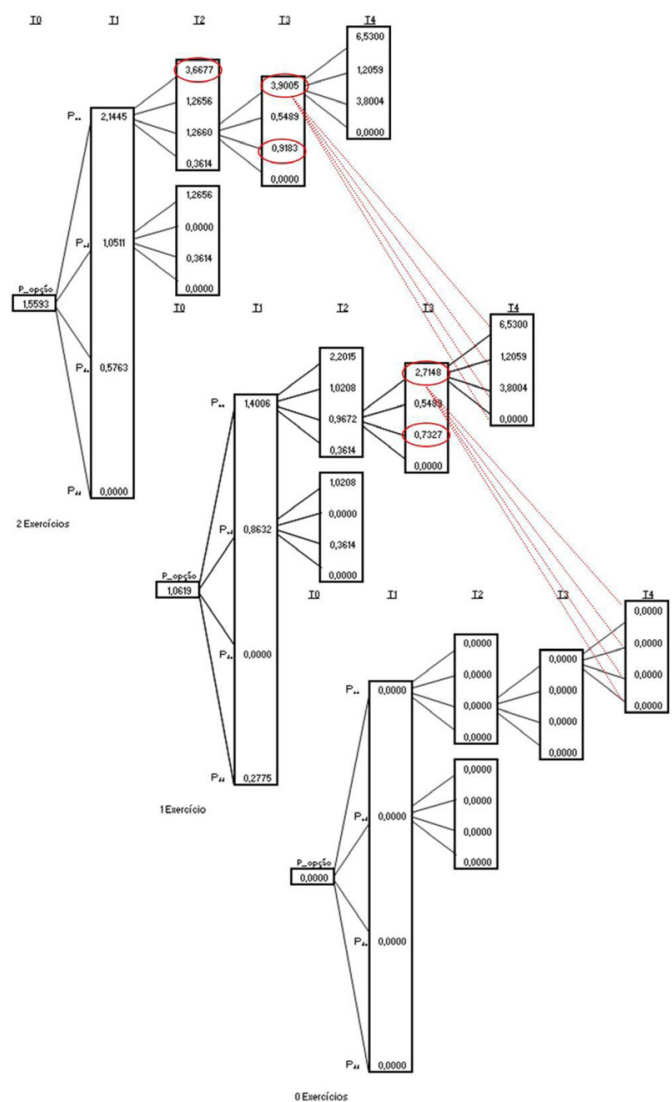

Figura 4. Árvore de opção de swing - preço de exercício fixo.

Tabela 3. Preços de exercício do GN.

\begin{tabular}{lc}
\hline Data de exercício & $\begin{array}{c}\text { Preço futuro } \\
\text { US\$/MMBtu }\end{array}$ \\
\hline Agosto de 2012 & 2,50 \\
Setembro de 2012 & 3,20 \\
Fevereiro de 2013 & 3,33 \\
Maio de 2013 & 3,40 \\
\hline
\end{tabular}

\section{Análise dos resultados}

Após incorporar sazonalidade e estimar os parâmetros do modelo através do filtro de Kalman, foi possível projetar os preços do GN para o período de vigência do contrato. A projeção desses preços foi feita com árvores binomiais bivariáveis, e as opções de swing nos contratos de GN foram calculadas seguindo a abordagem de Jaillet, Ronn e Tompaidis (2004).

No caso 1 em que o preço de exercício é fixo e igual a US\$3,70 por MMBtu, o valor da opção de swing encontrada foi de US\$1,56. Como em cada exercício podem ser contratados $2 \mathrm{MMBtus}$ além do contrato padrão, cada opção permite aumentar em 4MMBtus o volume. Tendo como base o preço à vista do GN na data de assinatura do contrato (US\$2,14 por MMBtu), o valor da opção de swing representa 73\% desse preço, o que equivale a 18\% do preço à vista do GN por MMBtu extra que pode ser contratado. Ou seja, em um mercado com incertezas de preço e demanda como é o de GN, o comprador está disposto a pagar $18 \%$ do preço à vista do GN por cada MMBtu adicional que ele tem o direito, mas não a obrigação de comprar em datas futuras.

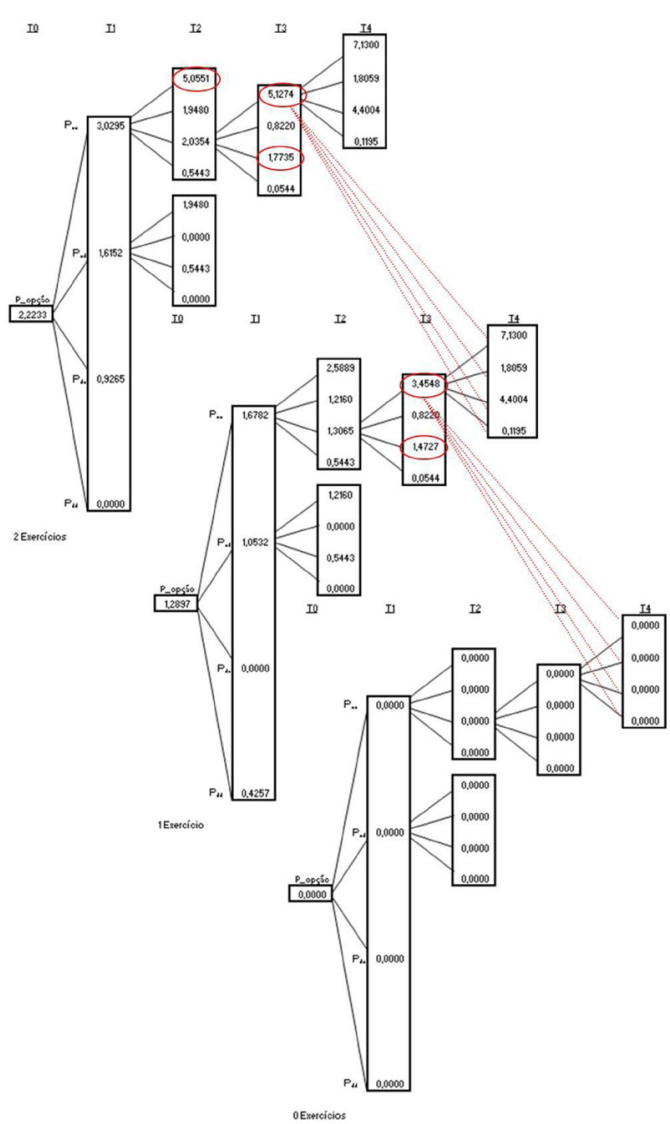

Figura 5. Árvore de opção de swing - preço de exercício variável. 
Caso queira assinar um contrato menos flexível e exercer uma vez o direito de comprar mais 2MMBtus, essa opção de swing vale US\$1,06, o que equivale a $50 \%$ do preço à vista do GN na data de assinatura do contrato. Nesse caso, paga-se $50 \%$ do preço do GN pela garantia de ter o fornecimento de 2 MMBtus adicionais. Esse valor equivale a $25 \%$ do preço à vista do GN por MMBtu extra que a opção lhe dá o direito de comprar.

No caso 2, em que o preço de exercício da opção de swing é variável e baseado na estrutura dos preços futuros nas diversas datas de exercício, o valor da opção de swing foi de US\$2,22, o que representa $104 \%$ do preço à vista do $\mathrm{GN}$, enquanto que o percentual por MMBtu é de 26\%. 0 valor da opção com a possibilidade de um único exercício é de US\$1,29 (60\% do preço do GN). Essa opção é mais valiosa que a opção de swing com preço de exercício fixo, pois no caso 2 os preços de exercício são todos inferiores a US $\$ 3,70$ (preço de exercício do contrato fixo), aumentando as chances de exercer a opção, o que a torna mais atrativa e valiosa.

\section{Conclusão}

0 crescimento do mercado de derivativos para commodities do setor de energia exige a necessidade de se desenvolver ferramentas eficientes na gestão de risco. 0 risco de mercado para essas commodities é uma variável estratégica relevante, por isso os derivativos tornaram-se um auxilio importante na administração dos riscos que surgem da alta volatilidade dos preços e incertezas no mercado.

Dentre estes derivativos, as opções exóticas permitem embutir flexibilidades nos contratos para proteger os agentes econômicos em cenários considerados críticos e incertos. Em muitos contratos, essas flexibilidades são embutidas por meio das opções de swing, que são opções exóticas que permitem o direito de receber volumes maiores do que o contratado. Uma opção de swing geralmente vem associada a um contrato padrão que garante ao seu detentor o fornecimento de um determinado volume de GN.

0 modelo de Schwartz e Smith (2000) com incorporação de sazonalidade trimestral foi utilizado para modelar e prever os preços do GN. Uma comparação desse modelo de dois fatores com um modelo de um fator, como o MGB, por exemplo, pode ser considerado objeto de alguma pesquisa futura.

0 apreçamento da opção foi efetuado seguindo o modelo de árvore binomial bivariável desenvolvido por Hahn e Dyer (2011). Em ambos os casos analisados, com preço de exercício fixo ou variável, o valor da opção de swing foi significativo, mostrando que essa opção deve ser incorporada nos contratos de GN. Em sintese, o estudo realizado indica que os contratos de GN devem levar em consideração as opções de swing, na medida em que as flexibilidades contratuais permitem maior proteção quanto às incertezas de preço e oscilações na demanda.

\section{Referências}

ADLAND, R.; JIA, H.; LU, J. Price dynamics in the market for Liquid Petroleum Gas transport. Energy Economics, v. 30, n. 3, p. 818-828, 2008. http://dx.doi.org/10.1016/j. eneco.2007.02.008

AlUBE, F. A. L. Modelos quantitativos em finanças com enfoque em commodities. Porto Alegre: Bookman, 2013.

AIUBE, F.; BAIDYA T.; TITO, E. Analysis of commodity prices with the particle filter. Energy Economics, v. 30, n. 2, p. 597-605, 2008. http://dx.doi.org/10.1016/j. eneco.2006.06.006

AGÊNCIA NACIONAL DE ENERGIA ELÉTRICA - ANEEL. Disponivel em: <http://www.aneel.gov.br>. Acesso em: 18 jan. 2012.

AGÊNCIA NACIONAL DO PETRÓLEO, GÁS NATURAL E BIOCOMBUSTIVEEIS - ANP. Indústria Brasileira de Gás Natural: Regulação Atual e Desafios Futuros. Rio de Janeiro: ANP, 2001. (Série ANP, n. 2).

BALDVINSDÓTTIR, E. K.; PALMBORG, L. On constructing a market consistent economic scenario generator. Sweden: Seminars Matematical Statistics KTH, 2011. PMid:22057365.

BANCO CENTRAL DO BRASIL. Ata do Compo, Maio/2012, $167^{\circ}$ Reunião, publicada em 8/06/2012. BCB, 2012.

BASTIAN-PINTO, C. L.; BRANDÃO, L. E. T.; HAHN, W. J. Flexibility as a source of value in the production of alternative fuels: The etanol case. Energy Economics, v. 31, n. 3, p. 411-422, 2009. http://dx.doi.org/10.1016/j. eneco.2009.02.004

BLACK, F.; SCHOLES, M. The pricing of options and corporate liabilities. Journal of Political Economy, v. 81, n. 3, p. 637-659, 1973. http://dx.doi.org/10.1086/260062

BOYLE, P. A. A Lattice Framework for option pricing with two state variables. Journal of Financial an Quantitative Analysis, v. 23, n. 1, p. 1-12, 1988. http://dx.doi. $\operatorname{org} / 10.2307 / 2331019$

BRENNAN, M. J.; SCHWARTZ, E. S. Evaluating natural resource investment. Journal of Business, v. 58, n. 2, p. 135-157, 1985. http://dx.doi.org/10.1086/296288

BRANDÃO, L. E. T.; DYER, J. S. Projetos de opções reais com incertezas correlacionadas. Base-Revista de Administração e Contabilidade da Unisinos, v. 6, n. 1, p. 19-26, 2009. http://dx.doi.org/10.4013/base.2009.61.02

BRANDÃO, L. E. T.; HAHN, W. J.; DYER, J. S. Using binomia decision trees to solve real options valuation problems. Decision Analysis, v. 2, n. 2, p. 69-88, 2005. http:// dx.doi.org/10.1287/deca.1050.0040

BRASIL. Ministério de Minas e Energia. Resenha Energética Brasileira (2011). Brasília: MME, 2011. 
BRIGO, D.; MERCURIO, F. Interest rate models - theory and practice with smile, inflation and credit. New York: Springer Finance - Verlag Berlin Heidelberg, 2010.

CARTEA, A.; WILLIAMS, T. UK Gas Market: the market price of risk and applications to multiple interruptible supply contracts. Energy Economics, v. 30, n. 3, p. 829-846, 2008. http://dx.doi.org/10.1016/j. eneco.2007.03.001

CLEWLOW, L.; STRICKLAND, C. Valuing energy options in a one factor model fitted to forward prices. Sydney: Work Paper, School of Finance and Economics, University of Technology, 1999.

CORTAZAR, G.; NARANJO, L. An N-factor gaussian model of oil futures prices. Journal of Futures Markets, v. 26, n. 2, p. 243-268, 2006. http://dx.doi.org/10.1002/fut.20198

COX, J. C.; ROSS, S. A.; RUBINSTEIN, M. Option Pricing: a simplified approach. Journal of Financial Economics, v. 7, n. 3, p. 229-263, 1979. http://dx.doi.org/10.1016/0304405X(79)90015-1

FlGUEROA, M. Pricing multiple interruptible-swing contracts. Birkbeck: University of London, Working Papers in Economics \& Finance, 2006.

GÁS NET. 0 site do gás natural. Disponível em: <http://www. gasnet.com.br>. Acesso em: 15 jan. 2012.

GIBSON, R.; SCHWARTZ, E. Stochastic convenience yield and pricing of oil contingent claims. Journal of Finance, v. 45, n. 3, p. 959-976, 1990. http://dx.doi. org/10.1111/j.1540-6261.1990.tb05114.x

GUiGuES, V.; SAGATIZABAL, C.; ZUBELll, J. P. Robust management and pricing of $L N G$ contracts with cancellation options. IMPA, 2010.

HAFF, I. H.; LINDQVIST, 0.; LøLAND, A. Risk Premium in the UK Natural Gas Forward Market. Energy Economics, v. 30, n. 5, p. 2420-2440, 2007. http://dx.doi.org/10.1016/j. eneco.2007.12.002

HAHN, W. J. A Discrete-time approach for valuing real options with underlying mean-reverting stochastic processes. 2005. Dissertation (Master's Degree)-University of Texas, Austin, 2005.

HAHN, W. J.; DYER, J. S. Discrete time modeling of meanreverting stochastic processes for real option valuation. European Journal of Operational Research, v. 183, n. 2, p. 534-548, 2008. http://dx.doi.org/10.1016/j. ejor.2006.11.015

HAHN, W. J.; DYER, J. S. A discrete time approach for modeling two-factor mean-reverting stochastic processes. Decision Analysis, v. 8, n. 3, p. 220-232, 2011. http://dx.doi.org/10.1287/deca.1110.0209

HAMBLY, B.; HOWISON, S.; KLUGE, T. Modelling spikes and pricing swing options in electricity markets. Quantitative Finance, v. 9, n. 8, p. 937-949, 2008. http://dx.doi. org/10.1080/14697680802596856

HARRISON, J.; KREPS, D. Martingales and arbitrage in multiperiod securities markets. Journal of Economic Theory, v. 20, n. 3, p. 381-408, 1979. http://dx.doi. org/10.1016/0022-0531(79)90043-7

HARRISON, J.; PLISKA, S. Martingales and stochastic integrals in the theory of continuous trading. Stochastic Processes and Their Applications, v. 11, p. 215-260, 1981. http:// dx.doi.org/10.1016/0304-4149(81)90026-0

HEM, O. D. et al. The option to switch from oil to natural gas in active offshore petroleum fields. In: ANNUAL INTERNATIONAL CONFERENCE REAL OPTIONS THEORY MEETS PRACTICE, 15., 2011, Turku. Proceedings... ROG, 2011.

HULL, J.; WHITE, A. Numerical procedures for implementing term structure models 1. Journal of Derivatives, v. 2, n. 1, p. 7-16, 1994a. http://dx.doi.org/10.3905/ jod.1994.407902

HULL, J.; WHITE, A. Numerical procedures for implementing term structure models Il. Journal of Derivatives, v. 2, n. 2, p. 37-42, 1994b. http://dx.doi.org/10.3905/ jod.1994.407908

JAILLET, P.; RONN, E. 1.; TOMPAIDIS, S. Valuation of Commodity-Based Swing Options. Management Science, v. 50, n. 7, p. 909-921, 2004. http://dx.doi.org/10.1287/ mnsc. 1040.0240

LUCIA, J. J.; SCHWARTZ, E. S. Electricity Prices and Power Derivatives: evidence from the Nordic Power Exchange. Review of Derivatives Research, v. 5, n. 1, p. 5-50, 2001. http://dx.doi.org/10.1023/A:1013846631785

MANOLIU, M.; TOMPAIDIS, S. Energy Futures Prices: Term Structure Models with Kalman Filter Estimation. Applied Mathematical Finance, v. 9, n. 1, p. 21-43, 2000. http:// dx.doi.org/10.1080/13504860210126227

MAROTTA, L. L. S. Calibração do Modelo de Schwartz-Smith com Filtro de Kalman. 2011. Dissertação (Mestrado em Métodos Matemáticos em Finanças)-Instituto Nacional de Matemática Pura e Aplicada, Rio de Janeiro, 2011.

MATHIAS, M. C. P. P. A formação da indústria global de gás natural: definição, condicionantes e desafios. 2008. Tese (Doutorado em Ciências em Planejamento Energético)Universidade Federal do Rio de Janeiro, Rio de Janeiro, 2008.

NELSON, D. B.; RAMASWAMY, K. Simple binomial processes as diffusion approximations $\mathrm{n}$ financial models. Review of Financial Studies, v. 3, n. 3, p. 393-430, 1990. http:// dx.doi.org/10.1093/rfs/3.3.393

PARK, F. C. Scenario analysis for bond and loan portfolios. Capital Markets \& Portfolio Research, Inc., 2003.

PARSONS, C. Valuing commodity storage contracts: a twofactor tree approach. WTM Energy Software, LLC, 2007.

PETROBRAS. Relatório de Atividades 2012. Rio de janeiro: Petrobras, 2012.

PINTO, C. L. B. Modelagem de opções reais com processos de reversão à média em tempo discreto: uma aplicação na Indústria Brasileira de Etanol. 2009. Tese (Doutorado)Pontifícia Universidade Católica do Rio de Janeiro, Rio de Janeiro, 2009.

SCHWARTZ, E. The stochastic behavior of commodity prices: implications for valuation and hedging. Journal of Finance, v. 52, n. 3, p. 923-973, 1997. http://dx.doi. $\operatorname{org} / 10.1111 /$ j.1540-6261.1997.tb02721.x

SCHWARTZ, E.; SMITH, J. Short-Term Variations and LongTerm Dynamics in Commodity Prices. Management Science, v. 46, n. 7, p. 893-911, 2000. http://dx.doi. org/10.1287/mnsc.46.7.893.12034 
SIMÕES, M. D. P. et al. Opções de Swing no Mercado Brasileiro de Energia Elétrica. Revista de Economia e Administração, v. 10, n. 4, p. 591-610, 2011.

SØRENSEN, C. Modeling Seasonality in Agricultural Commodity Futures. Journal of Futures Markets, v. 22, n. 5, p. 393-426, 2002. http://dx.doi.org/10.1002/ fut. 10017
TSENG, C.; LIN, K. A Framework Using Two-Factor Price Lattices for Generation Asset Valuation. Operations Research, v. 55, n. 2, p. 234-251, 2007. http://dx.doi. org/10.1287/opre.1060.0355

ZHANG, B.; OOSTERLEE, C. W. An efficient pricing algorithm for swing options based on fourier cosine expansions. Reports of the Department of Applied Mathematical Analysis, Delft University of Technology, The Netherlands, 2010.

\section{Pricing swing options embedded in natural gas contracts using a two-factor model}

\section{Abstract}

In the natural gas (NG) market, contracts incorporate flexibility in the volume of the product. These contracts are known as swing options. Such contracts allow the option holder to exercise the right to receive greater or smaller amounts of NG contracted in accordance with market. Variation in the price of NG, which is the main source of uncertainty, was modeled in this study as a stochastic process using the two-factor model of Schwartz and Smith (2000), which was incorporated into assessment of the quarterly seasonality. To estimate NG spot prices using the Henry Hub prices of futures contracts traded on the NYMEX, it was necessary to implement the Kalman filter. The pricing of the option was conducted using the binomial tree model bi-variable developed by Hahn and Dyer (2011). The value of the swing option was positive in both cases analyzed, indicating that this option should be considered for inclusion in NG contracts. The characteristics of the analysis were the same as those specified in Jaillet, Ronn and Tompaidis (2004).

\section{Keywords}

Finance. Derivatives. Contracts pricing. Stochastic processes. 